\title{
Mediators of the Relationship Between Attachment and Dispositional Mindfulness in Adolescents
}

\author{
Karen Goodall $^{1,2}$ (D) Zara P. Brodie $^{3}$ - Matthias Schwannauer ${ }^{1}$ \\ Published online: 16 May 2020 \\ (C) The Author(s) 2020
}

\begin{abstract}
Objectives Converging studies have confirmed a robust relationship between attachment and mindfulness, leading to the proposition that dispositional mindfulness originates from early interpersonal relationships. Despite this assertion, few studies have examined these relationships in adolescents and young adults. Theoretical linkages between mindfulness, attachment, relationships, and self-regulation suggest that attachment may be linked to mindfulness via self-regulatory capacity. This study examined the direct effect of attachment on dispositional mindfulness in adolescents and young adults, as well as indirect effects via attentional control and emotion regulation.

Methods Participants were 421 adolescents and young people age $12-24$ years $(M=17, \mathrm{SD}=4.10)$. They completed a selfreport survey measuring attachment anxiety, attachment avoidance, dispositional mindfulness, attentional control (AC), and emotion regulation (ER).

Results There was a moderate effect of attachment anxiety $95 \%$ CI [ - 1.45, -0.93$]$, and a small effect of attachment avoidance $95 \%$ CI $[-1.27,-0.44]$ on mindfulness. Attachment anxiety was indirectly related to mindfulness via AC, 95\% CI [-0.32, - 10], and ER, [-0.80, - 0.41], as was attachment avoidance (AC, 95\% CI [-44, - 0.10], ER, 95\% CI [-0.52, - 0.19]. Serial mediation analyses revealed serial paths from AC through ER and ER through AC with small effect sizes relative to individual mediators. Conclusions Levels of dispositional mindfulness in adolescents were linked to attachment insecurity dimensions through attention control and emotion regulation. Attachment dimension-specific differences were noted; for the anxiety dimension, emotion regulation exerts a stronger mediating effect.
\end{abstract}

Keywords Dispositional mindfulness $\cdot$ Attachment $\cdot$ Attentional control $\cdot$ Emotion regulation $\cdot$ Age

Individual variation in dispositional mindfulness has been related to a range of salutary benefits, such as enhanced emotion processing and regulation, reduced rumination, and lower levels of depressive symptoms (Tomlinson et al. 2018). As a result, research examining the correlates of dispositional mindfulness has burgeoned recently (Rau and Williams 2016). Attachment theory has provided a useful theoretical

Karen Goodall

karen.goodall@ed.ac.uk

1 Department of Clinical and Health Psychology, University of Edinburgh, Edinburgh, Scotland

2 Department of Clinical Psychology, University of Edinburgh, Edinburgh, Scotland

3 School of Media, Culture and Society, University of the West of Scotland, Paisley, Scotland framework for explaining individual differences in naturally occurring mindfulness, with a recent meta-analysis confirming moderate mean effect sizes between measures of attachment and dispositional mindfulness (Stevenson et al. 2017). These studies have been pivotal to the assertion that the capacity to be mindful has its roots in early interpersonal relationships (Ryan et al. 2007; Shaver et al. 2007). Despite claims about the developmental antecedents of dispositional mindfulness, it is unclear whether similar associations between attachment and dispositional mindfulness can be detected in adolescents and young adults.

Intrinsic to the proposition that dispositional mindfulness arises from interpersonal interactions is the conceptual overlap between attachment, self-regulatory capacity, and mindfulness (Caldwell and Shaver 2013; Goodall et al. 2012). Individual differences in attentional control and emotion regulation have been associated both with attachment patterns (Gillath et al. 2009; Pascuzzo et al. 2015) and with dispositional 
mindfulness (Guendelman et al. 2017; Malinowski 2013). Arguably, adolescence is a period of consolidation of both attachment representations and self-regulatory processes (Ahmed et al. 2015; Allen et al. 2004). Therefore, investigation of the correlates and mediators of dispositional mindfulness during this period is warranted.

Mindfulness can be conceptualized as comprising three self-regulatory elements: cognitive and emotional flexibility which are facilitated by attention regulation (Malinowski 2013). Mindfulness and self-regulation are likely to have a bi-directional relationship. Sustained mindfulness practice leads to neurobiological changes associated with improved capacity across these areas in children and adults (Guendelman et al. 2017; Kaunhoven and Dorjee 2017). At the same time, higher dispositional mindfulness has been associated with higher levels of attentional capacity (Ruocco and Direkoglu 2013), lower cortical emotional reactivity (Brown et al. 2012), and enhanced emotion regulation capacity (Modinos et al. 2010). Mindfulness may, therefore, be as much a product of self-regulatory ability as a predictor of it (Masicampo and Baumeister 2007).

The capacity to regulate attention and emotion has also increasingly been associated with attachment-based individual differences. According to attachment theory, individuals, who have a history of sensitive and contingent caregiving, internalize a model of psychological security which enables them to engage in flexible and open attentional control (Gillath et al. 2009; Muris and Dietvorst 2006). By contrast, insecurely attached individuals engage in habitual and automatic attentional strategies that protect against rejection and shame (Mikulincer et al. 2003). Although automatic, these strategies are cognitively demanding (Gillath et al. 2009) and theoretically reduce the capacity to engage in mindful awareness.

Attachment is commonly measured on two dimensions of insecurity: attachment anxiety and attachment avoidance (Brennan et al. 1998), with lower scores denoting security. As these dimensions have been associated with distinct regulatory styles, the attachment framework further enables investigations of specific regulatory pathways. The attachment anxiety dimension, characterized by a compulsive need to guard against rejection or abandonment (Shaver and Mikulincer 2002), has been associated with a high arousal/low control style of regulation. This encompasses selective, threatfocused attention monitoring (Ein-Dor et al. 2011b), high subjective and neurological arousal in response to negative emotion (Rognoni et al. 2008), and compulsive rumination (Wei et al. 2005). The attachment avoidance dimension has been associated with a hypoactivating regulatory style that defends against information that threatens the sense of an invulnerable self. This is characterized by the use of suppressive strategies that limit attention to emotional input (Liu et al. 2017), low affective evaluation (Vrtička and Vuilleumier 2012), and blocking of negative emotion (Ein-Dor et al. 2011a). A small number of studies have demonstrated that attachment dimensions are linked to mindfulness indirectly through selfregulatory capacity, including attentional control and emotion regulation (Caldwell and Shaver 2013; Pepping et al. 2013). Whether or not these mediating paths are relevant for adolescent populations is unclear.

The period of adolescence, spanning from 10 to 24 years, encompasses significant neurobiological and social-relational changes (Sawyer et al. 2018). Firstly, executive functions, subserved by cortical reactivity and control, are developing during adolescence, leading to increased attentional and emotion regulation capacity (Ahmed et al. 2015; Bunge and Wright 2007) and age-related differences in emotion regulation repertoires (Zimmermann and Iwanski 2014). Although attachment patterns are relatively stable across adolescence and adulthood, they can change in response to environmental changes which overwhelm the adolescent's capacity for affect regulation (Allen et al. 2004; Pinquart et al. 2013). The metaregression of Stevenson et al. (2017) indicated that age did not moderate the relationship between attachment and mindfulness. However, participants in this study were 16 years or older. Thus, it remains unclear whether mechanisms seen in adult samples are a reflection of consolidated attachment representations and self-regulatory processes, or whether they can also be detected in an adolescent sample.

In this study, we investigated the direct and indirect relationships between attachment and dispositional mindfulness via attentional control and emotion regulation in adolescents from 12 to 24 years. Based on previous research, we predicted small to moderate effect sizes for both dimensions of attachment insecurity. As a preliminary investigation, we tested for a moderating effect of age before advancing to mediation analysis. Based on the meta-regression of Stevenson et al. (2017), we predicted that there would be no conditional effect of age on the relationship. We further investigated potential mediators of these relationships. Based on previous studies, we predicted a different pattern of mediating effects for the attachment dimensions. We predicted a stronger indirect effect of attentional control than emotion regulation in the relationship between attachment avoidance and mindfulness, due to the suppressive attention tendencies associated with this dimension. Based on the high arousal/low control associated with the anxiety dimension, we predicted that the largest indirect effect on mindfulness would be via emotion regulation. Attentional control and emotion regulation are, arguably, interlinked. For example, attentional capacity has been linked to emotion awareness and clarity (O'Bryan et al. 2017; Teper et al. 2013), and conversely emotional arousal can affect attentional capacity (Jeffries et al. 2008). We therefore explored serial indirect pathways from attentional control to emotion regulation and vice versa. In view of a lack of relevant literature, we advanced no predictions about these serial pathways. 


\section{Method}

\section{Participants}

Three of the 424 individuals, who completed the survey, were excluded due to not meeting the age criteria. The remaining 421 participants were age 12 to 24 years $\left(M_{\mathrm{age}}=17\right.$ years, SD = 4.10). The majority $(87 \%)$ were UK residents ( $4 \%$ otherEuropean-other and $9 \%$ from other countries) and $61 \%$ were female. A small proportion of participants $(11 \%)$ indicated that they mediated weekly or more frequently. An independent samples $t$ test was conducted to compare mindfulness scores for those who indicated that they mediated on a weekly or more frequent basis and those who meditate less frequently or not at all. There was no significant difference in scores between meditators $(M=32.79, \mathrm{SD}=6.26)$ and non-meditators, $t(419)=0.95, p=0.34,95 \%$ CI $[-0.88,2.52],(M=$ $31.96, \mathrm{SD}=5.51$. Thus, all participants were retained in the sample. Based on previous research, path sizes were estimated for the $a$ and $b$ paths. Following the recommendations of Fritz and MacKinnon (2007), the sample size required to achieve a small-medium mediation effect ( $\alpha=0.26, \beta=0.39$ ), using percentile bootstrapping, was 126 .

\section{Procedures}

This study was approved by the University Ethics Committee and the Local Education Authority of the relevant schools. Inclusion criteria included age 12-24 years old with good understanding of written English. Two hundred and fiftyfive participants, age 12-18 years, were recruited from three state schools. The remainder of the sample was contacted via university email and social media. Informed consent was obtained from all individual participants included in the study. Additional parental consent was obtained for participants under 16 years of age. The majority of the participants accessed the survey via a secure online survey platform. For school-age participants, this was completed during school hours. One hundred and thirty-three school-age participants completed a paper version.

\section{Measures}

\section{Mindfulness}

The Cognitive and Affective Mindfulness Scale-Revised (CAMS-R; Feldman et al. 2007) is a unidimensional, 12-item, self-report measure, designed to reflect the breadth of the mindfulness concept while retaining simple language. It is the only mindfulness measure which has been normed for use with children and adolescents. Participants rated the extent to which items applied to them (e.g., "It is easy for me to concentrate on what I am doing") on a scale of 1 (rarely/not at all) to 4 (almost always). Conceptually, it differs from other mindfulness scales in measuring a willingness and capacity to be mindful ('I try to notice my thoughts without judging them') rather than the extent to which one is mindful during the day (Bergomi et al. 2012). It is therefore based on components proposed to be required to reach a mindful state (attention, awareness, present focus, and acceptance/nonjudgment). The scale has good convergent validity with other mindfulness measures, significant associations with measures of distress, well-being and emotion regulation, and good internal consistency, $\alpha=0.76$ (Feldman et al. 2007).

\section{Attachment}

The Experiences in Close Relationships-Relationship Structures Scale (ECR-RS; Fraley et al. 2011) is a 9-item questionnaire which assesses two-dimensional attachment structures: attachment anxiety and attachment avoidance. Participants were asked to indicate how well statements, such as "I am afraid that this person may abandon me," represented their feelings on a scale of 1 (strongly disagree) to 7 (strongly agree). Higher scores denote greater attachment insecurity. The questionnaire exhibits satisfactory construct validity, including factor-specific links to the model of adult attachment (Bartholomew and Horowitz 1991), and supports the application of the ECR-RS to assessing relationship-specific adolescent attachment structures (Donbaek and Elklit 2014). Reported internal consistency for the dimensions ranges from 0.81 to 0.92 (Fraley et al. 2011).

\section{Emotion regulation}

The Difficulties in Emotion Regulation Scale (DERS; Gratz and Roemer 2004) is a 36-item self-report tool, measuring difficulties in emotion regulation. Participants endorsed statements on a scale of 1 (never) to 5 (almost always). The scale comprises a total score, as well as 6 sub-scales: lack of emotional awareness, lack of emotional clarity, impulse control, goal-directed behavior (remaining goal-focused when distressed), non-acceptance of emotional responses (being distressed by negative emotions), and limited access to emotion regulation strategies. Gratz and Roemer (2004) report high internal consistency, $\alpha=0.93$ for the total scale used in this study. The scale also has good reliability and validity with adolescents (Weinberg and Klonsky 2009).

\section{Attentional control}

The Attentional Control Scale (ACS; Derryberry and Reed 2002) is a 20-item self-report measure of executive control over attention. Statements, such as "It's very hard for me to concentrate on a difficult task when there are noises around," were endorsed on a scale of 1-4, (almost never) to 4 (always). 
Reported internal consistency for the total score is high $(\alpha=$ 0.75; Muris et al. 2007). Several studies have begun to provide support for the predictive validity of the ACS (Derryberry and Reed 2002).

\section{Data Analyses}

Data analyses were conducted using SPSS version 23. Inspection of the raw data revealed no missing values. Descriptive statistics were computed for all study variables. Pearson's correlation coefficients were inspected to assess associations between variables based on Cohen's (1988) criteria for small (0.10), medium (0.30), and large (0.50) associations. In all models reported, when attachment anxiety was the antecedent variable, attachment avoidance was entered as a control variable and vice versa.

Using multiple linear regression analysis, mindfulness was regressed onto both dimensions of attachment insecurity to assess the unique variance that each dimension explained. To examine the potential interacting effect of age on the relationship between attachment and mindfulness, a moderating mediation analysis was conducted using process model 1 of the SPSS macro PROCESS (Version 3) (Hayes 2018).

To test whether attentional control and emotion regulation mediated the relationship between attachment and mindfulness, parallel multiple mediation analyses (PMMA) were conducted using PROCESS model 4 (see Fig. 1). A defining feature of PMMAs is that no mediator is modelled as influencing another mediator in the model. This model allows for estimation of a simultaneous test of both mediators, while accounting for shared variance between them. We hypothesized that attachment insecurity (attachment anxiety and attachment avoidance $(X)$ ) would indirectly influence mindfulness $(Y)$ via attentional control $\left(M_{1}\right)$ and emotion regulation $\left(M_{2}\right)$.

In a PMMA, while mediators can be correlated, it is assumed that no mediator casually influences another. To further probe whether the mediators impact on one another in a

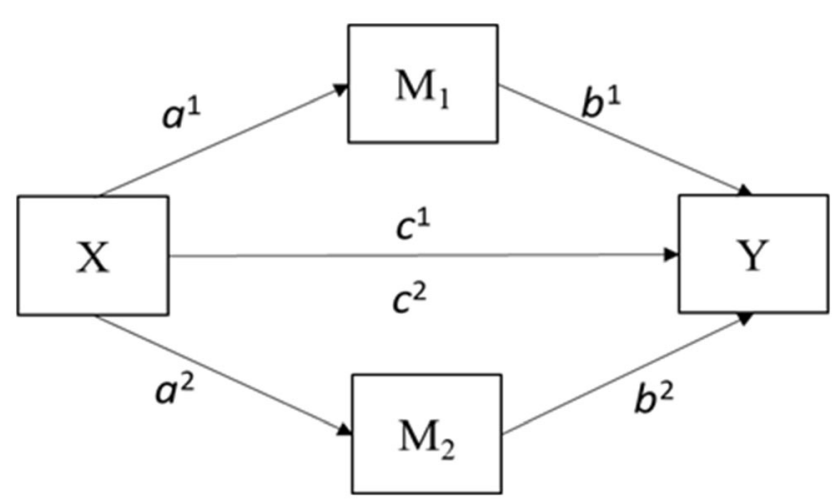

Fig. 1 Parallel Multiple Mediation Model (PMM) serial manner, we conducted serial multiple mediation analyses (SMMA), using PROCESS model 6 (Fig. 2). In SMMAs, the assumption of no causal relationship between two mediators is rejected a priori (Hayes 2018). SMMAs facilitate modelling of direct and indirect effects, including a serial indirect effect via attentional control through emotion regulation. We modelled this separately for both attachment dimensions and reversed the order of the mediators in two additional models. Thus, four serial multiple mediator models were conducted.

In all models, pairwise comparison between specific indirect effects determined whether any indirect effect was statistically different from any other in the model. All regression/ path coefficients were reported in unstandardized forms. A bootstrapping method was used as it does not assume normal distributions for variables. Resampling was done 10,000 times as recommended by Hayes (2018).

\section{Results}

\section{Descriptive statistics and correlations}

Table 1 shows the correlations, means, standard deviations, and alpha coefficients for all study variables, indicating high internal consistency of measures and relationships in the expected direction. Medium negative correlations emerged between dispositional mindfulness and attachment anxiety, $r=-$ $0.44, p<0.001$; attachment avoidance, $r=-0.28, p<0.001$; attentional control, $r=0.54, p<0.001$; and difficulties in emotion regulation, $r=0.62, p<0.001$. Age was not significantly correlated with mindfulness scores but had a small significant positive correlation with attachment anxiety, $r=$ $0.11, p=0.02$.

\section{Relationships between attachment dimensions and mindfulness}

Table 2 displays the results of regressing dispositional mindfulness scores onto attachment anxiety and avoidance simultaneously. Attachment insecurity explained $23 \%$ of the unique variance in dispositional mindfulness, $F(2$, $\left.418)=60.95, p<0.001, R^{2}=0.23\right)$. The standardized coefficients indicate a moderate effect size for anxiety, $\beta$ $=-0.40,95 \% \mathrm{CI}[-1.45,-0.93]$, and a small effect size for avoidance, $\beta=-0.18,95 \%$ CI $[-1.27,-0.44]$. This confirms that each attachment dimension is a unique predictor of dispositional mindfulness when the effect of the other dimension is controlled. For each unit increase in attachment anxiety and avoidance, dispositional mindfulness scores decreased by 1.19 and 0.86 units respectively. 
Fig. 2 Serial Multiple Mediation Model (SMM)

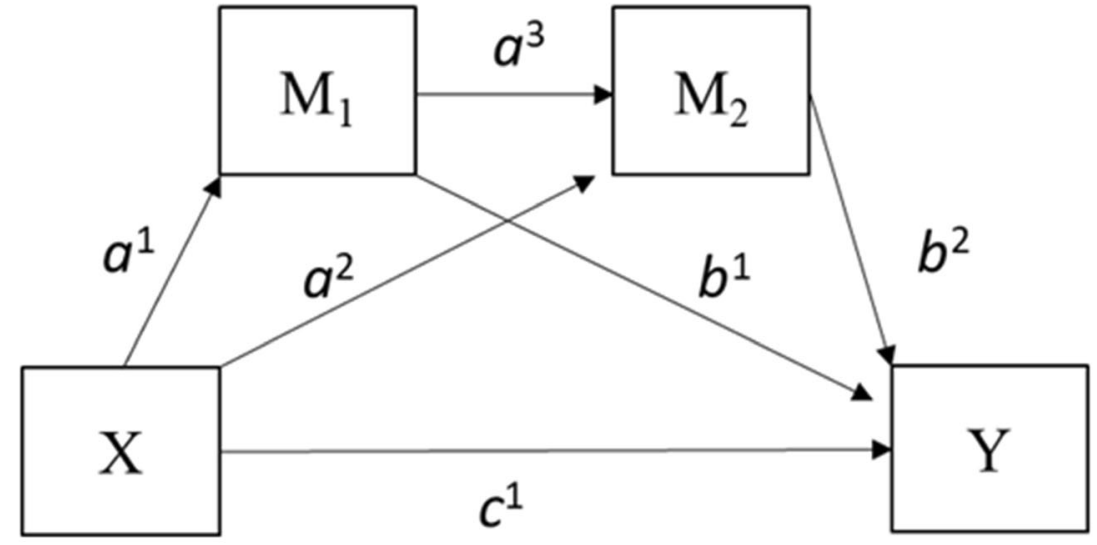

\section{Moderating effect of age on the relationship between attachment and mindfulness}

To examine whether there was an effect of age on the relationship between attachment and mindfulness, two moderation analyses were conducted using the PROCESS macro. In the first model, mindfulness was regressed on attachment anxiety, with age as a moderator. The model was significant, $F(4$, $416)=32.80, p<0.001$. Attachment anxiety did not explain a significant proportion of unique variance in mindfulness when attachment avoidance and age were controlled, $b=-0.15$, SE $=0.55,95 \%$ CI $[-0.93,1.22]$. Although there was a small interaction effect between age and attachment anxiety, $b=-$ $0.08, \mathrm{SE}=0.03,95 \% \mathrm{CI}[-0.14,-0.02]$, the conditional effect was significant at lower $(b=-0.84, \mathrm{SE}=0.19,95 \%$ CI $[-1.21,-0.48]$, mean $(b=-1.16, \mathrm{SE}=0.13,95 \% \mathrm{CI}[-$ $1.42,-0.90]$, and older ages $(b=-1.48, \mathrm{SE}=0.18,95 \% \mathrm{CI}$ $[-1.83,-1.12]$, and the Johnson-Neyman technique indicated that there were no statistical transition points within the observed age range. Thus, while there was a small moderating effect, a specific age range of statistical change could not be determined from the sample range.

In the second model, with attachment avoidance as the independent variable, the model was also significant, $F(4$, $416)=31.37, p<0.001$. Attachment avoidance explained a significant proportion of unique variance in dispositional mindfulness when attachment anxiety and age were controlled, $b=-2.03, \mathrm{SE}=0.89,95 \%$ CI $[-3.78,-0.28]$. There was no interaction effect, however, indicating that the relationship between attachment avoidance and mindfulness does not vary by age $(b=-0.07, \mathrm{SE}=0.05,95 \% \mathrm{CI}[-0.03$, 0.16 ).

\section{Mediating effects}

To examine the direct and indirect effects of attachment on mindfulness, via attentional control and emotion regulation, two parallel multiple mediator analyses were conducted (Table 3). In all models henceforth, age was entered as a control variable. In the first model, the total effect of attachment anxiety on mindfulness was significant, $b=-1.17,95 \%$ CI $[-1.43,-0.91]$. The direct effect was also significant, $b=$ $-0.39,95 \%$ CI $[-0.65,-0.13]$, indicating that attachment anxiety explains additional variance over that explained by the mediators. The unstandardized regression coefficients indicated that attentional control, $b=-0.20,95 \% \mathrm{CI}[-0.32,-0.10]$ and emotion regulation, $b=-0.58,95 \%$ CI $[-0.80,-0.41])$ were significant mediators of this relationship. Pairwise comparison of the specific indirect effects confirmed significant differences, $b=-0.39,95 \%$ CI $[-0.61,-0.16])$. The hypothesis that emotion regulation would be a stronger mediator of this relationship than attentional support was supported.
Table 1 Correlations, means, standard deviations, and internal reliability of study variables

\begin{tabular}{lllllllll}
\hline Variable & 1 & 2 & 3 & 4 & 5 & Mean & SD & $\alpha$ \\
\hline 1 CAMS-R & 1 & & & & & 31.97 & 5.51 & 0.77 \\
2 ECR-anxiety & $-0.44^{* *}$ & 1 & & & 4.13 & 1.83 & 0.80 \\
3 ECR-avoidance & $-0.28^{* *}$ & $0.25^{* *}$ & 1 & & & 3.54 & 1.16 & 0.89 \\
4 ACS & $-0.54^{* *}$ & $-0.25^{* *}$ & $-0.23^{* *}$ & 1 & & 18.55 & 4.42 & 0.80 \\
5 DERS & $-0.62^{* *}$ & $0.49^{* *}$ & $0.22^{* *}$ & $-0.35^{* *}$ & 1 & 90.11 & 24.38 & 0.94 \\
6 Age & -0.08 & $0.11^{*}$ & -0.06 & 0.06 & $-0.62 * *$ & 17.01 & 0.20 & - \\
\hline
\end{tabular}

$C A M S-R$ Cognitive and Affective Mindfulness Scale-Revised, ECR Experiences in Close Relationships-Revised Questionnaire, ACS Attentional Control Scale, DERS Difficulties in Emotion Regulation Scale. $* * p<0.001$, * $p<$ 0.05 
Table 2 Regression model of mindfulness on attachment

\begin{tabular}{lllllll}
\hline Source & $B$ & $\mathrm{SE}$ & $\beta$ & $t$ & $p$ & $95 \% \mathrm{CI}$ \\
\hline Intercept & 39.79 & 0.84 & & 47.12 & 0.00 & {$[38.13,41.45]$} \\
ECR-anxiety & -1.19 & 0.13 & -0.40 & -8.97 & 0.00 & {$[-1.45,-0.93]$} \\
ECR-avoidance & -0.86 & 0.21 & -0.18 & -4.04 & 0.00 & {$[-1.27,-0.44]$} \\
\hline
\end{tabular}

In the second model, the total effect of attachment avoidance on mindfulness was also significant, $b=-0.88,95 \% \mathrm{CI}$ $[-1.30,-0.46]$. The direct effect of attachment avoidance on mindfulness was not significant, $b=-0.29,95 \%$ CI $[-0.64$, 0.07], indicating that the effect of attachment avoidance on mindfulness is fully accounted for by the mediators. There were significant indirect effects via attentional control, $b=-$ $0.26,95 \%$ CI $[-0.44,-0.10]$ and emotion regulation, $b=-$ $0.34,95 \%$ CI $[-0.52,-0.19]$. Pairwise comparison of the indirect effect found no significant differences in the indirect effects of attentional control and emotion regulation, $b=-$ $0.08,95 \%$ CI $[-27,0.13]$. The hypothesis that attentional control would be a stronger mediator in the relationship between attachment avoidance and mindfulness was therefore not supported.

\section{Serial mediations}

Serial mediation hypothesizes a causal linking chain of mediators. We tested two possible mediating pathways linking attachment to mindfulness: attentional control to emotion regulation and the reverse path. Separate models were conducted, with attachment anxiety and avoidance as antecedent variables. This led to a total of four models (Table 4). All models were compared in terms of the significant paths yielded by different causal models of the mediators (Table 5).

In SMMA1, there were three significant paths linking attachment anxiety to mindfulness: a serial path to attentional control to emotion regulation, $b=-0.09,95 \%$ CI $[-0.15$, -

Table 3 PMM analyses with emotion regulation and attentional control as mediators

\begin{tabular}{lllll}
\hline IV & Path & Effect & SE & $95 \%$ CI \\
\hline ECR-anxiety & Total effect $(c)$ & -1.17 & 0.13 & {$[-1.43,-0.91]$} \\
& Direct effect $\left(c^{\prime}\right)$ & -0.39 & 0.13 & {$[-0.65,-0.13]$} \\
& Total indirect effect & -0.78 & 0.11 & {$[-1.00,-0.58]$} \\
& Indirect via ACS & -0.20 & 0.05 & {$[-0.32,-0.10]$} \\
& Indirect via DERS & -0.58 & 0.10 & {$[-0.80,-0.41]$} \\
ECR-avoidance & Total effect (c) & -0.88 & 0.21 & {$[-1.30,-0.46]$} \\
& Direct effect $\left(c^{\prime}\right)$ & -0.29 & 0.18 & {$[-0.64,0.07]$} \\
& Total indirect effect & -0.60 & 0.14 & {$[-0.86,-0.33]$} \\
& Indirect via ACS & -0.26 & 0.09 & {$[-0.44,-0.10]$} \\
& Indirect via DERS & -0.34 & 0.08 & {$[-0.52,-0.19]$} \\
\hline
\end{tabular}

0.05], as well as indirect paths through attentional control, $b=$ $-0.20,95 \% \mathrm{CI}[-0.31,-0.10]$, and emotion regulation, $b=-$ $0.50,95 \%$ CI [ $-0.69,-0.35]$. Pairwise comparison of indirect effects found significant differences between all pathways, indicating that emotion regulation was a stronger mediator than the serial path or attentional control. In SMMA 2, there were three significant paths linking attachment avoidance to mindfulness: a serial path to attentional control to emotion regulation, $b=-0.12$, 95\% CI $[-0.21,-0.05]$, and paths via attentional control, $b=-0.26,95 \%$ CI $[-0.44,-0.11]$, and emotion regulation, $b=-0.22,95 \%$ CI $[-0.38,-0.10]$. Pairwise comparison of indirect effects indicated that the effect of attentional control was stronger than that of the serial path, $b=-0.14,95 \%$ CI $[-0.30,-0.04]$.

SMMA3 and SMMA4 tested the same models, with the order of the mediators reversed. In SMMA3, emotion regulation to attentional control was a significant serial path in the relationship between attachment anxiety and mindfulness, $b=$ $-0.27,95 \%$ CI $[-0.38,-0.19])$. There was also a significant indirect effect of emotion regulation, $b=-0.58,95 \% \mathrm{CI}[-$ $0.79,-0.41]$. In SMMA4, attachment avoidance was indirectly associated with mindfulness through a significant serial pathway from emotion regulation to attentional control, $b=$ $-0.03,95 \%$ CI $[-0.05,-0.02]$, and a significant path via emotion regulation, $b=-0.07,95 \%$ CI $[-0.10,-0.04]$. Pairwise comparison of indirect effects in SMMA3 and 4 confirmed that the effect of the serial path was significantly weaker than that of emotion regulation.

The results from the SMMA analyses indicate that emotion regulation and attentional control may interact bi-directionally in mediating the relationship between attachment insecurity and mindfulness. Comparison of the significant serial paths also suggests that there were subtle differences in the mechanisms linking the two attachment dimensions to mindfulness. Comparison of SMMA1 and SMMA3 suggests that the most plausible serial path linking attachment anxiety to mindfulness is one where the dysregulated emotion associated with attachment anxiety limits attentional control and, thus, the capacity to be mindful. By contrast, comparing SMMA 2 and 4, suggests that the plausible serial path linking attachment avoidance to mindfulness is characterised by attentional control difficulties negatively impacting emotion regulation capacity and subsequently mindfulness.

\section{Discussion}

This study aimed to extend our current understanding of the relationship between attachment and dispositional mindfulness, by focusing on an adolescent and young adult sample. The present study also assessed direct and indirect paths from attachment to mindfulness through examining attentional control and emotion regulation as parallel and serial mediators. 
Table 4 Possible serial models, accounting for mindfulness, according to different causal orders

\begin{tabular}{llll}
\hline SMM Model & IV & $\mathrm{M}^{1}$ & $\mathrm{M}^{2}$ \\
\hline 1 & ECR-anxiety $\rightarrow$ & Attentional control $\rightarrow$ & Emotion regulation $\rightarrow$ \\
2 & ECR-avoidance $\rightarrow$ & Attentional control $\rightarrow$ & Emotion regulation $\rightarrow$ \\
3 & ECR-anxiety $\rightarrow$ & Emotion regulation $\rightarrow$ & Attentional control $\rightarrow$ \\
4 & ECR-avoidance $\rightarrow$ & Emotion regulation $\rightarrow$ & Attentional control $\rightarrow$ \\
\hline
\end{tabular}

Consistent with previous research on adult samples (Stevenson et al. 2017), we found a moderate negative association between attachment anxiety and mindfulness and a small negative association between attachment avoidance and mindfulness. In contrast to previous research (Stevenson et al. 2017), we found a small moderating effect of age on the relationship between attachment anxiety and mindfulness. This suggests that the negative relationship between attachment anxiety and mindfulness increases over time. Consistent with our hypotheses, we found that attentional control and emotion regulation mediated the relationship between attachment and dispositional mindfulness. Furthermore, subtle differences were noted in the mediating pathways linking attachment dimensions (anxiety and avoidance) to mindfulness.

Converging studies on adult participants have pointed to a robust pattern of association between individual dimensions of attachment insecurity and mindfulness levels. The results of this study affirm that the same pattern of association can be detected in a sample of adolescents and young adults, despite the documented changes in attachment representations and self-regulatory capacity that are characteristic of this life stage. Extending the age range to a younger sample provides further support for the view that dispositional mindfulness is related to early interpersonal relationships. This study also had methodological strengths in testing the effects of prior meditation experience prior to the main analyses and controlling for covariance between the attachment dimensions. In contrast with the meta-regression conducted by Stevenson et al. (2017), which comprised studies on mainly adult participants, the present study found a stronger effect of attachment anxiety on mindfulness with increasing age. Further replication of this effect is required before strong conclusions can be drawn.

The results of the present study build on previous studies which have modelled mediating pathways and have identified attentional control and emotion regulation as mechanisms between attachment and mindfulness (e.g., Caldwell and Shaver 2013; Pepping et al. 2013). In this study, we used parallel models to test each mediator while accounting for the shared variance between them. As expected, both variables mediated relationships between attachment dimensions and mindfulness. Based on research that asserts that individual attachment dimensions are associated with distinct style of self-regulation, we expected to see subtle differences in the mediating paths between each dimension and mindfulness. This was partly supported by results. We hypothesized that there would be a stronger mediating effect of attentional control than emotion regulation in the relationship between attachment avoidance and mindfulness. In fact, the mediating effect of attentional control was not statistically different to that of emotion regulation. This suggests that the characteristic regulatory
Table 5 SMM analyses with emotion regulation and attentional control as mediators

\begin{tabular}{llllll}
\hline Model & IV & Path & $b$ & SE & $95 \%$ CI \\
\hline 1 & ECR-anxiety & Indirect total & 0.78 & 0.11 & {$[1.00,-0.58]$} \\
& Indirect via ACS & -0.20 & 0.05 & {$[-0.31,-0.10]$} \\
& Indirect via DERS & -0.50 & 0.08 & {$[-0.69,-0.35]$} \\
& Indirect via ACS $\rightarrow$ DERS & -0.09 & 0.03 & {$[-0.15,-0.05]$} \\
& Indirect total & -0.60 & 0.13 & {$[-0.87,-0.35]$} \\
& ECR-avoidance & Indirect via ACS & -0.26 & 0.08 & {$[-0.44,-0.11]$} \\
& Indirect via DERS & -0.22 & 0.07 & {$[-0.38,-0.10]$} \\
& Indirect via ACS $\rightarrow$ DERS & -0.12 & 0.04 & {$[-0.21,-0.05]$} \\
& ECR-anxiety & Indirect total & -0.78 & 0.11 & {$[-1.02,-0.59]$} \\
& Indirect via ACS & -0.07 & 0.05 & {$[-0.02,0.16]$} \\
& Indirect via DERS & -0.58 & 0.10 & {$[-0.79,-0.41]$} \\
& Indirect via DERS $\rightarrow$ ACS & -0.27 & 0.05 & {$[-0.38,-0.19]$} \\
& ECR-avoidance & Indirect total & -0.60 & 0.13 & {$[-0.86,-0.35]$} \\
& Indirect via ACS & -0.02 & 0.01 & {$[-0.05,0.00]$} \\
& Indirect via DERS & -0.07 & 0.02 & {$[-0.10,-0.04]$} \\
& & Indirect via DERS $\rightarrow$ ACS & -0.03 & 0.01 & {$[-0.05,-0.02]$} \\
& & & &
\end{tabular}


style associated with the avoidance dimension reduces the capacity to be mindful through emotion regulation facets, such as poor awareness and clarity of emotions (Goodall 2015), as well as maladaptive attentional strategies (Mikulincer et al. 2003). The hypothesis that there would be a stronger mediating effect of emotion regulation than attentional control in the relationship between attachment anxiety and mindfulness was supported. Thus, while both attentional control and difficulties in regulating emotion are mechanisms linking attachment dimensions to mindfulness, for people with high attachment anxiety, emotion dysregulation is the predominant mechanism. Attachment anxiety has been associated with high levels of arousal in response to negative external or internal stimuli and a paucity of adaptive strategies for managing arousal (Rognoni et al. 2008; Wei et al. 2005), leading to inflexible modes of processing that are "mindless" rather than mindful.

We also conducted serial mediation analyses to determine whether the mediators linked attachment to mindfulness in a casual chain. We made no assumptions about order and tested the serial path from attentional control to emotion regulation and the reverse path. Modelling alternative serial indirect effects avoided false assumptions based on unidirectional serial models. Notably, all four serial paths were significant, indicating that attentional control and emotion regulation are likely to have a bi-directional effect on each other in linking attachment to mindfulness. The largest serial path linking attachment avoidance to mindfulness was via attentional control to emotion regulation. From a mindfulness perspective, attending to thoughts and emotions is a first step in increasing the clarity and awareness required for detached observation (Baer 2003). The serial path therefore offers an additional path from attachment avoidance to mindfulness, in conjunction with attentional control and emotion regulation as parallel mediators. These serial models are particularly worth commenting on in relation to the attachment anxiety dimension. The largest serial path linking attachment anxiety to mindfulness was a small to moderate serial path via emotion regulation to attentional control. In this model, attentional control exerted an effect only through this serial path. This model supports the high arousal/low control that has been associated with attachment anxiety. In anxiously attached individuals, dysregulated emotional states are associated with rumination and a difficulty in disengaging from negative thoughts (Gillath et al. 2005; O'Bryan et al. 2017). Attention is therefore turned inwards, reducing the capacity to engage in open monitoring and detached observation.

Self-regulation of attentional control is proposed to be the basis for cognitive and emotional flexibility and is often given a central role in mindfulness training (Malinowski 2013). However, for anxiously attached individuals, self-regulation of arousal may be of more importance than self-regulation of attention in understanding pathways to dispositional mindfulness. This proposition is supported by the results of a recent factor analytic study which suggests that attachment anxiety may be particularly associated with lower mindfulness through low use of emotion re-appraisal strategies (Stevenson et al. 2019). The findings are illuminating in relation to the conceptualization of dispositional mindfulness, as they advance our understanding of specific mechanisms likely to link attachment-based representations to individual differences in dispositional mindfulness.

\section{Limitations and Future Research Directions}

As with all research, the study has some limitations. The first concerns the reliance on a self-report measure of mindfulness. It has been noted that the validity of self-report mindfulness measures may be hampered by how items are understood semantically by participants (Grossman 2011). The second is the use of a unidimensional mindfulness scale. Utilizing a multidimensional scale would have supported investigation of mediating pathways between attachment and distinct facets of mindfulness. Third, the use of multiple-item scales within a single survey may lead to common methods bias, where spurious correlations can result from participant characteristics, such as transient mood states, dispositional affectivity, or a desire to respond logically and consistently (Podsakoff et al. 2003). Future research could control for common methods bias by conducting multitrait-multimethod studies, where participants are measured on constructs using multiple methods or instruments (Bagozzi and Yi 1993). Finally, while this study provided novel insight into the relationship between attachment and mindfulness in adolescents and adults, crosssectional studies, do not provide evidence of a temporal relationship. Longitudinal data would be required to confirm the causal nature of the concepts investigated and cross-lagged panel analysis is warranted.

Similarly, mediation analyses do not denote causal relationships. Randomized control trials would be warranted to determine the impact of improved attentional or emotion regulation skills on the relationship between attachment and mindfulness. As the relationship between attachment anxiety and mindfulness was moderated by age, the role of age should be considered in future studies investigating the relationship between these concepts.

Authors' Contribution KG: designed and executed the study, analyzed the data, and wrote the paper. ZB: collaborated with the data analyses and the writing of the study. MS: collaborated in the writing and editing of the final manuscript.

Data availability All data are available at the Open Science Framework (https://osf.io/ 6t7c4/).

\section{Compliance with Ethical Standards}

Conflict of Interest The authors declare that they have no conflict of interest. 
Ethical Approval All procedures have been approved by the Health in Social Science research ethics committee (Ref: CLIN671) at the University of Edinburgh and have been performed in accordance with the ethical standards laid down in the 1964 Declaration of Helsinki and its later amendments.

Informed consent Informed consent was obtained from all individual participants included in the study.

Open Access This article is licensed under a Creative Commons Attribution 4.0 International License, which permits use, sharing, adaptation, distribution and reproduction in any medium or format, as long as you give appropriate credit to the original author(s) and the source, provide a link to the Creative Commons licence, and indicate if changes were made. The images or other third party material in this article are included in the article's Creative Commons licence, unless indicated otherwise in a credit line to the material. If material is not included in the article's Creative Commons licence and your intended use is not permitted by statutory regulation or exceeds the permitted use, you will need to obtain permission directly from the copyright holder. To view a copy of this licence, visit http://creativecommons.org/licenses/by/4.0/.

\section{References}

Ahmed, S. P., Bittencourt-Hewitt, A., \& Sebastian, C. L. (2015). Neurocognitive bases of emotion regulation development in adolescence. Developmental Cognitive Neuroscience, 15, 11-25. https:// doi.org/10.1016/j.den.2015.07.006.

Allen, J. P., McElhaney, K. B., Kuperminc, G. P., \& Jodl, K. M. (2004). Stability and change in attachment security across adolescence. Child Development, 75(6), 1792-1805. https://doi.org/10.1111/j. 1467-8624.2004.00817.x.

Baer, R. A. (2003). Mindfulness training as a clinical intervention: a conceptual and empirical review. Clinical Psychology: Science and Practice, 10, 125-143. https://doi.org/10.1093/clipsy.bpg015.

Bagozzi, R. P., \& Yi, Y. (1993). Multitrait-multimethod matrices in consumer research: critique and new developments. Journal of Consumer Psychology, 2(2), 143-170. https://doi.org/10.1016/ S1057-7408(08)80022-8.

Bartholomew, K., \& Horowitz, L. M. (1991). Attachment styles among young adults: a test of a four-category model. Journal of Personality and Social Psychology, 61(2), 226-244. https://doi.org/10.1037/ 0022-3514.61.2.226.

Bergomi, C., Tschacher, W., \& Kupper, Z. (2012). The Assessment of mindfulness with self-report measures: existing scales and open issues. Mindfulness, 4(3), 191-202. https://doi.org/10.1007/s12671012-0110-9.

Brennan, K. A., Clark, C. L., \& Shaver, P. R. (1998). Self-report measurement of adult romantic attachment: an integrative overview. In J. A. Simpson \& W. S. Rholes (Eds.), Attachment theory and close relationships (pp. 46-76). Guilford Press.

Brown, K. W., Goodman, R. J., \& Inzlicht, M. (2012). Dispositional mindfulness and the attenuation of neural responses to emotional stimuli. Social Cognitive and Affective Neuroscience, 8(1), 93-99. https://doi.org/10.1093/scan/nss004.

Bunge, S. A., \& Wright, S. B. (2007). Neurodevelopmental changes in working memory and cognitive control. Current Opinion in Neurobiology, 17(2), 243-250. https://doi.org/10.1016/j.conb. 2007.02.005.

Caldwell, J. G., \& Shaver, P. R. (2013). Mediators of the link between adult attachment and mindfulness. Interpersona, 7(2), 299-310. https://doi.org/10.5964/ijpr.v7i2.133.
Cohen, J. (1988). Statistical power analysis for the behavioral sciences (2nd ed.). Lawrence Erlbaum Associates.

Derryberry, D., \& Reed, M. A. (2002). Anxiety-related attentional biases and their regulation by attentional control. Journal of Abnormal Psychology, 111(2), 225-236. https://doi.org/10.1037//0021-843x. 111.2.225.

Donbaek, D. F., \& Elklit, A. (2014). A validation of the experiences in close relationships-relationship structures scale (ECR-RS) in adolescents. Attachment \& Human Development, 16(1), 58-76. https://doi. org/10.1080/14616734.2013.850103.

Ein-Dor, T., Mikulincer, M., \& Shaver, P. R. (2011a). Attachment insecurities and the processing of threat-related information: studying the schemas involved in insecure people's coping strategies. Journal of Personality and Social Psychology, 101(1), 78-93. https://doi.org/10.1037/a0022503.

Ein-Dor, T., Mikulincer, M., \& Shaver, P. R. (2011b). Effective reaction to danger: attachment insecurities predict behavioral reactions to an experimentally induced threat above and beyond general personality traits. Social Psychological and Personality Science, 2(5), 467-473. https://doi.org/10.1177/1948550610397843.

Feldman, G., Hayes, A., Kumar, S., Greeson, J., \& Laurenceau, J. P. (2007). Mindfulness and emotion regulation: the development and initial validation of the Cognitive and Affective Mindfulness ScaleRevised (CAMS-R). Journal of Psychopathology and Behavioral Assessment, 29(3), 177-190. https://doi.org/10.1007/s10862-0069035-8.

Fraley, R. C., Heffernan, M. E., Vicary, A. M., \& Brumbaugh, C. C. (2011). The Experiences in Close Relationships - Relationship Structures questionnaire: a method for assessing attachment orientations across relationships. Psychological Assessment, 23(3), 615625. https://doi.org/10.1037/a0022898.

Fritz, M. S., \& MacKinnon, D. P. (2007). Required sample size to detect the mediated effect. Psychological Science, 18(3), 233-239. https:// doi.org/10.1111/j.1467-9280.2007.01882.x.

Gillath, O., Bunge, S. A., Shaver, P. R., Wendelken, C., \& Mikulincer, M. (2005). Attachment-style differences in the ability to suppress negative thoughts: exploring the neural correlates. Neuroimage, 28(4), 835-847. https://doi.org/10.1016/j.neuroimage.2005.06.048.

Gillath, O., Giesbrecht, B., \& Shaver, P. R. (2009). Attachment, attention, and cognitive control: attachment style and performance on general attention tasks. Journal of Experimental Social Psychology, 45(4), 647-654. https://doi.org/10.1016/j.jesp.2009.02.011.

Goodall, K. (2015). Individual differences in the regulation of positive emotion. The role of attachment and self-esteem. Personality and Individual Differences, 74, 208-213. https://doi.org/10.1016/j.paid. 2014.10.033.

Goodall, K., Trejnowska, A., \& Darling, S. (2012). The relationship between dispositional mindfulness, attachment security, and emotion regulation. Personality and Individual Differences, 52, 622626. https://doi.org/10.1016/j.paid.2011.12.008.

Gratz, K. L., \& Roemer, L. (2004). Multidimensional assessment of emotion regulation and dysregulation: development, factor structure, and initial validation of the difficulties in emotion regulation scale. Journal of Psychopathology and Behavioral Assessment, 26(41), 41-54. https://doi.org/10.1023/B:JOBA.0000007455.08539.94.

Grossman, P. (2011). Defining mindfulness by how poorly I think I pay attention during everyday awareness and other intractable problems for psychology's (re)invention of mindfulness. Comment on Brown et al. (2011). Psychological Assessment, 23, 1034-1040. https://doi. org/10.1037/a0022713.

Guendelman, S., Medeiros, S., \& Rampes, H. (2017). Mindfulness and emotion regulation: insights from neurobiological, psychological, and clinical studies. Frontiers in Psychology, 8, 220. https://doi. org/10.3389/fpsyg.2017.00220. 
Hayes, A. (2018). Introduction to mediation, moderation and conditional process analysis: a regression-based approach (2nd ed). The Guildford Press.

Jeffries, L. N., Smilek, D., Eich, E., \& Enns, J. T. (2008). Emotional valence and arousal interact in attentional control. Psychological Science, 19(3), 290-295. https://doi.org/10.1111/j.1467-9280. 2008.02082.x.

Kaunhoven, R. J., \& Dorjee, D. (2017). How does mindfulness modulate self-regulation in pre-adolescent children? An integrative neurocognitive review. Neuroscience and Biobehavioral Reviews, 74, 163-184. https://doi.org/10.1016/j.neubiorev.2017.01.007.

Liu, Y., Ding, Y., Lu, L., \& Chen, X. (2017). Attention bias of avoidant individuals to attachment emotion pictures. Scientific Reports, 7 , 41631. https://doi.org/10.1038/srep41631.

Malinowski, P. (2013). Neural mechanisms of attentional control in mindfulness meditation. Frontiers in Neuroscience, 7, 8. https:// doi.org/10.3389/fnins.2013.00008.

Masicampo, E. J., \& Baumeister, R. F. (2007). Relating mindfulness and self-regulatory processes. Psychological Inquiry, 18(4), 255-258. https://doi.org/10.1080/10478400701598363.

Mikulincer, M., Shaver, P. R., \& Pereg, D. (2003). Attachment theory and affect regulation: the dynamics, development and cognitive consequences of attachment-related strategies. Motivation and Emotion, 27(2), 77-102. https://doi.org/10.1023/A:1024515519160.

Modinos, G., Ormel, J., \& Aleman, A. (2010). Individual differences in dispositional mindfulness and brain activity involved in reappraisal of emotion. Social Cognitive and Affective Neuroscience, 5(4), 369377. https://doi.org/10.1093/scan/nsq006.

Muris, P., \& Dietvorst, R. (2006). Underlying personality characteristics of behavioral inhibition in children. Child Psychiatry and Human Development, 36(4), 437-445. https://doi.org/10.1007/s10578-0060014-9.

Muris, P., Meesters, C., \& Rompelberg, L. (2007). Attention control in middle childhood: relations to psychopathological symptoms and threat perception distortions. Behaviour Research and Therapy, 45, 997-1010. https://doi.org/10.1016/j.brat.2006.07.010.

O'Bryan, E. M., Kraemer, K. M., Johnson, A. L., McLeish, A. C., \& McLaughlin, L. E. (2017). Examining the role of attentional control in terms of specific emotion regulation difficulties. Personality and Individual Differences, 108, 158-163. https://doi.org/10.1016/j. paid.2016.12.015.

Pascuzzo, K., Moss, E., \& Cyr, C. (2015). Attachment and emotion regulation strategies in predicting adult psychopathology. SAGE Open. https://doi.org/10.1177/2158244015604695.

Pepping, C. A., Davis, P. J., \& O’Donovan, A. (2013). Individual differences in attachment and dispositional mindfulness: The mediating role of emotion regulation. Personality and Individual Differences, 54(3), 453-456. https://doi.org/10.1016/j.paid.2012.10.006.

Pinquart, M., Feußner, C., \& Ahnert, L. (2013). Meta-analytic evidence for stability in attachments from infancy to early adulthood. Attachment \& Human Development, 15(2), 189-218. https://doi. org/10.1080/14616734.2013.746257.

Podsakoff, P. M., MacKenzie, S. B., Lee, J. Y., \& Podsakoff, N. P. (2003). Common method biases in behavioral research: a critical review of the literature and recommended remedies. Journal of Applied Psychology, 88(5), 879-903. https://doi.org/10.1037/ 0021-9010.88.5.879.

Rau, H. K., \& Williams, P. G. (2016). Dispositional mindfulness: a critical review of construct validation research. Personality and Individual Differences, 93, 32-43. https://doi.org/10.1016/j.paid. 2015.09.035.
Rognoni, E., Galati, D., Costa, T., \& Crini, M. (2008). Relationship between adult attachment patterns, emotional experience and EEG frontal asymmetry. Personality and Individual Differences, 44(4), 909-920. https://doi.org/10.1016/j.paid.2007.10.021.

Ruocco, A., \& Direkoglu, E. (2013). Delineating the contributions of sustained attention and working memory to individual differences in mindfulness. Personality and Individual Differences, 54(2), 226230. https://doi.org/10.1016/j.paid.2012.08.037.

Ryan, R. M., Brown, K. W., \& Creswell, J. D. (2007). How integrative is attachment theory? Unpacking the meaning and significance of felt security. Psychological Inquiry, 18(3), 177-182. https://doi.org/10. 1080/10478400701512778.

Sawyer, S., Azzopardi, P. S., Wickremarathne, D., \& Patton, G. C. (2018). The age of adolescence. The Lancet Child \& Adolescent Health, 2, 223-228. https://doi.org/10.1016/S2352-4642(18) 30022-1.

Shaver, P. R., \& Mikulincer, M. (2002). Attachment-related psychodynamics. Attachment and Human Development, 4, 133-161. https:// doi.org/10.1080/14616730210154171.

Shaver, P. R., Lavy, S., Saron, C. D., \& Mikulincer, M. (2007). Social foundations of the capacity for mindfulness: an attachment perspective. Psychological Inquiry, 18, 264-271. https://doi.org/10.1080/ 10478400701598389.

Stevenson, J. C., Emerson, L. M., \& Millings, A. (2017). The relationship between adult attachment orientation and mindfulness: a systematic review and meta-analysis. Mindfulness, 8(6), 1438-14558. https:// doi.org/10.1007/s12671-017-0733-y.

Stevenson, J. C., Millings, A., \& Emerson, L. (2019). Psychological wellbeing and coping: the predictive value of adult attachment, dispositional mindfulness, and emotion regulation. Mindfulness, 10, 256271. https://doi.org/10.1007/s12671-018-0970-8.

Teper, R., Segal, Z. V., \& Inzlicht, M. (2013). Inside the mindful mind: how mindfulness enhances emotion regulation through improvements in executive control. Current Directions in Psychological Science, 22(6), 449-454. https://doi.org/10.1177/ 0963721413495869

Tomlinson, E. R., Yousaf, O., Vittersø, A. D., \& Jones, L. (2018). Dispositional mindfulness and psychological health: a systematic review. Mindfulness, 9(1), 23-43. https://doi.org/10.1007/s12671017-0762-6.

Vrtička, P., \& Vuilleumier, P. (2012). Neuroscience of human social interactions and adult attachment style. Frontiers in Human Neuroscience, 6, 212. https://doi.org/10.3389/fnhum.2012.00212.

Wei, M., Vogel, D. L., Ku, T. Y., \& Zakalik, R. A. (2005). Adult attachment, affect regulation, negative mood, and interpersonal problems: the mediating roles of emotional reactivity and emotional cutoff. Journal of Counseling Psychology, 52(1), 14-24. https://doi.org/ 10.1037/0022-0167.52.1.14.

Weinberg, A., \& Klonsky, E. (2009). Measurement of emotion dysregulation in adolescents. Psychological Assessment, 21, 616-621. https://doi.org/10.1037/a0016669.

Zimmermann, P., \& Iwanski, A. (2014). Emotion regulation from early adolescence to emerging adulthood and middle adulthood: age differences, gender differences, and emotion-specific developmental variations. International Journal of Behavioral Development, 38(2), 182-194. https://doi.org/10.1177/0165025413515405.

Publisher's Note Springer Nature remains neutral with regard to jurisdictional claims in published maps and institutional affiliations. 